\title{
GENERIC EXPANDING MAPS WITHOUT ABSOLUTELY CONTINUOUS INVARIANT $\sigma$-FINITE MEASURE
}

\author{
Artur Avila ANd Jairo Bochi*
}

\begin{abstract}
We show that a $C^{1}$-generic expanding map of the circle has no absolutely continuous invariant $\sigma$-finite measure.
\end{abstract}

\section{Introduction}

If $f$ is a measurable transformation of a Lebesgue measure space $(X, \mathcal{A}, \lambda)$ to itself, that does not preserve the measure $\lambda$, one can study the invariant measures of $f$ and compare them to $\lambda$. An especially interesting case is when $f$ is non-singular with respect to $\lambda$ (in the sense that $\lambda(A)=0$ iff $\lambda\left(f^{-1}(A)\right)=0$ ), but nevertheless there exist no $\sigma$-finite invariant measure which is absolutely continuous with respect to $\lambda$. Such maps $f$ are called of type III (with respect to the measure). Their existence was conjectured by Halmos [4] and established by Ornstein [12]. Other examples were given later; let us cite a few (when not specified, the relevant measure is Riemannian):

- certain piecewise linear homeomorphisms of the circle, by Herman [7];

- certain $C^{\infty}$-diffeomorphisms of the circle, by Katznelson [9];

- a $C^{\infty}$ non-invertible map of the 2-torus, by Hawkins and Silva [6];

- the full shift on 2 symbols, with respect to some product measure, by Hamachi [5].

- a $C^{1}$ expanding map of the circle (constructed using Hamachi's example), by Bruin and Hawkins [2].

Recall that $C^{1+\alpha}$ expanding maps have absolutely continuous invariant probability measures, so the regularity of the example of Bruin and Hawkins is essentially sharp.

The question of whether the absence of aci $\sigma$ (absolutely continuous invariant $\sigma$ finite measure) is actually a generic (in the usual topological sense) phenomenon for $C^{1}$ expanding maps of the circle seems to have been first posed by Quas [13]. Later investigations [3] indicated that the known methods failed to decide the question either way. It was also known that $C^{1}$-generic maps do behave "pathologically" in some respects (they have no absolutely continuous invariant probability measure [13]), but not in others (they are ergodic and conservative with respect to Lebesgue measure [13], and they possess a unique physical measure [3]).

In this paper we show that the type III property is indeed $C^{1}$-generic for expanding maps of the circle.

We also mention that the non-existence of finite invariant measures that are absolutely continuous with respect to Riemannian measure was shown to be a generic

Received by the editors October 19, 2006.

*Beneficiário de auxílio financeiro CAPES-Brasil 
property among (expanding or not) $C^{1}$ maps of compact manifolds of any dimension - see $[1]$.

Of course, it is natural to ask whether the result of the present paper is still true for expanding maps on higher dimension. It is not clear whether our methods can be extended.

Concerning non-necessarily expanding maps of a compact manifold, there are $C^{1}$ open sets of transformations that do have some absolutely continuous $\sigma$-finite invariant measure (maps with a sink, for instance). In this regard, we ask whether a $C^{1}$-generic map has no absolutely continuous $\sigma$-finite invariant measure which is conservative (all w.r.t. Riemannian measure). We will show that this is true at least for onedimensional maps, see corollary 1.

Let us now give the precise statements.

Let $\mathbb{T}^{1}=\mathbb{R} / \mathbb{Z}$ be the circle. Let $\mathcal{E}^{1}$ be the set of all $C^{1}$ maps $f: \mathbb{T}^{1} \rightarrow \mathbb{T}^{1}$ which are expanding, i.e.,

$$
\exists c>0, \exists \lambda>1 \text { s.t. }\left|\left(f^{n}\right)^{\prime}(x)\right|>c \lambda^{n} \forall x \in \mathbb{T}^{1}, \forall n \in \mathbb{N} .
$$

We endow the set $\mathcal{E}^{1}$ with the $C^{1}$ topology. Let $m$ denote the Lebesgue measure on $\mathbb{T}^{1}$ normalized so that $m\left(\mathbb{T}^{1}\right)=1$. We say that a $\sigma$-finite measure on $\mathbb{T}^{1}$ is an acio for a map $f: \mathbb{T}^{1} \rightarrow \mathbb{T}^{1}$ if it is absolutely continuous with respect to $m$ and it is $f$-invariant. Our main result is:

Theorem 1. There exists a residual set $\mathcal{R} \subset \mathcal{E}^{1}$ such that if $f \in \mathcal{R}$ then $f$ has no acio.

Corollary 1. Let $X$ be either the circle $\mathbb{T}^{1}$ or the compact interval $[0,1]$. There exists a residual set $\mathcal{R}^{\prime}$ of the space of $C^{1}(X, X)$ such that if $f \in \mathcal{R}^{\prime}$ then $f$ has no conservative acio.

Proof. Hyperbolic maps form an open and dense subset $\mathcal{H}$ of $C^{1}(X, X)$ by [8]. (See also [10] for the recent extension to higher regularity.) The map that associates to $f \in \mathcal{H}$ its non-wandering set $\Omega(f)$ is upper semi-continuous in the Hausdorff topology. Moreover, if $f \in \mathcal{H} \cap C^{2}(X, X)$ then $m(\Omega(f))=0$ unless $X=\mathbb{T}^{1}$ and $f$ is expanding, see [11]. It follows that generically, either $m(\Omega(f))=0$ or $f \in \mathcal{E}^{1}$. In the first case, $f$ cannot have a conservative aci $\sigma$ (since any conservative measure must be supported on the non-wandering set). In the second case, generically there is no aci $\sigma$ at all, by theorem 1.

\section{Some preliminaries}

A reduction. Let $\mathcal{E}_{0}^{1}$ be the subset of $\mathcal{E}^{1}$ consisting of maps $f$ satisfying $f(0)=0$ and such that (1) holds with $c=1$. We will actually prove:

Theorem 2. There exists a residual set $\mathcal{R}_{0} \subset \mathcal{E}_{0}^{1}$ such that if $f \in \mathcal{R}_{0}$ then $f$ has no acio.

Let us show that theorem 2 implies theorem 1 . Let $f \in \mathcal{E}^{1}$, and let $p_{f}$ be a fixed point of $f$. Then for $\tilde{f}$ in a small open neighborhood $\mathcal{U}$ of $f$ in $\mathcal{E}^{1}$, there exists a unique fixed point $p_{\tilde{f}}$ of $\tilde{f}$ near $p_{f}$, moreover this fixed point depends continuously 
on $\tilde{f}$. Let $n \geq 1$ be such that $\left|\left(f^{n}\right)^{\prime}(x)\right|>1$ for every $x \in \mathbb{T}^{1}$. Define

$$
h(x)=\sum_{k=0}^{n-1}\left|\left(f^{k}\right)^{\prime}(x)\right| \quad \text { so that } \quad \int_{\mathbb{T}^{1}} h(x) d x=\frac{\left|d_{f}\right|^{n}-1}{\left|d_{f}\right|-1},
$$

where $d_{f}$ is the degree of $f$. Let $H_{\tilde{f}}$ be the orientation preserving $C^{1}$ diffeomorphism of $\mathbb{T}^{1}$ such that $H_{\tilde{f}}\left(p_{\tilde{f}}\right)=0$ and $H_{\tilde{f}}^{\prime}(x)=\frac{\left|d_{f}\right|-1}{\left|d_{f}\right|^{n}-1} h(x)$. Then $H_{\tilde{f}}$ depends continuously on $\tilde{f} \in \mathcal{E}^{1}$. Define a map $g=H_{f} \circ f \circ H_{f}^{-1}$. For any $x \in \mathbb{T}^{1}$, writing $y=H_{f}^{-1}(x)$, we have

$$
\left|g^{\prime}(x)\right|=\frac{h(f(y)) \cdot\left|f^{\prime}(y)\right|}{h(y)}=\frac{h(y)+\left|\left(f^{n}\right)^{\prime}(y)\right|-1}{h(y)}>1 .
$$

Hence $g \in \mathcal{E}_{0}^{1}$. Shrinking $\mathcal{U}$ to a smaller open neighborhood of $f$ if necessary, we see that $\tilde{g}=H_{\tilde{f}} \circ \tilde{f} \circ H_{\tilde{f}}^{-1}$ belongs to $\mathcal{E}_{0}^{1}$ for every $\tilde{f} \in \mathcal{U}$. Consider the mapping $\Pi: \tilde{f} \mapsto \tilde{g}$; it is clearly continuous. It is also open: for any $\hat{g} \in \mathcal{E}_{0}^{1}$ close to $g, H_{f}^{-1} \circ \hat{g} \circ H_{f} \in \mathcal{E}^{1}$ is close to $f$ and is mapped by $\Pi$ to $\hat{g}$. The preimage of $\mathcal{R}_{0}$ under $\Pi$ is thus a residual subset of $\mathcal{U}$, which contains only maps which have no aci $\sigma$.

Lispchitz maps. Let $\mathcal{E}_{0}^{\text {lip }}$ be the set of Lipschitz local homeomorphisms $f: \mathbb{T}^{1} \rightarrow \mathbb{T}^{1}$, such that $f(0)=0$ and

$$
\lambda_{f}=\underset{x \in \mathbb{T}^{1}}{\operatorname{essinf}}\left|f^{\prime}(x)\right|>1 .
$$

We consider $\mathcal{E}_{0}^{\text {lip }}$ endowed with the topology induced from the Lipschitz metric:

$$
d_{\text {lip }}(f, g)=\underset{x \in \mathbb{T}^{1}}{\operatorname{ess} \sup }\left|f^{\prime}(x)-g^{\prime}(x)\right| .
$$

We also let

$$
\Lambda_{f}=\underset{x \in \mathbb{T}^{1}}{\operatorname{ess} \sup }\left|f^{\prime}(x)\right|
$$

The distortion of the restriction of some iterate of $f$ to some interval is

$$
\operatorname{Dist}\left(f^{n} \mid J\right)=\frac{\operatorname{ess~sup}_{x \in J}\left|\left(f^{n}\right)^{\prime}(x)\right|}{\operatorname{essinf}_{x \in J}\left|\left(f^{n}\right)^{\prime}(x)\right|} .
$$

Clearly, if $f^{n} \mid J$ is 1-1 onto $I$ then for every measurable $X \subset J$ of positive measure,

$$
\frac{1}{\operatorname{Dist}\left(f^{n} \mid J\right)} \leq \frac{m\left(f^{n}(X)\right) / m(I)}{m(X) / m(J)} \leq \operatorname{Dist}\left(f^{n} \mid J\right)
$$

Recurrence properties. We say that $f \in \mathcal{E}_{0}^{\text {lip }}$ is ergodic with respect to $m$ if every measurable set $X$ such that $f^{-1}(X)=X$ satisfies $m(X)=0$ or $m(X)=1$. We say that $f \in \mathcal{E}_{0}^{\text {lip }}$ is conservative with respect to $m$ if every measurable set $X$ such that $X \cap \bigcup_{k=1}^{\infty} f^{-k}(X)=\varnothing$ satisfies $m(X)=0$.

It is easy to see that $f \in \mathcal{E}_{0}^{\text {lip }}$ is ergodic and conservative with respect to $m$ if and only if for every measurable set $X$ which is forward invariant (that is, $f(X) \subset X$ ) we have $m(X)=0$ or $m(X)=1$.

If $f \in \mathcal{E}_{0}^{\text {lip }}$ and $X \subset \mathbb{T}^{1}$ is a measurable set, then we denote $X_{f}$ the set of points in $X$ that return to $X$ by forward iteration by $f$. It is easy to see that if $f$ is conservative then $m\left(X_{f}\right)=m(X)$.

We denote by $f_{X}: X_{f} \rightarrow X$ the first return map. 
Lemma 1. If $\mu$ is any $f$-invariant $\sigma$-finite measure then $\mu\left(f_{X}^{-1} Y\right) \leq \mu(Y)$ for all measurable $Y \subset X$.

Proof. We can assume $\mu(Y)<\infty$. For $n \geq 1$, let

$$
Z_{n}=f^{-1}\left(X^{c}\right) \cap \cdots \cap f^{-(n-1)}\left(X^{c}\right) \cap f^{-n}(Y), \quad Y_{n}=X \cap Z_{n} .
$$

Then $\bigsqcup_{n=1}^{\infty} Y_{n}=f_{X}^{-1}(Y)$. Since $Z_{n+1}=f^{-1}\left(X^{c} \cap Z_{n}\right)$, we have

$$
\mu\left(Y_{n}\right)=\mu\left(X \cap Z_{n}\right)=\mu\left(Z_{n}\right)-\mu\left(X^{c} \cap Z_{n}\right)=\mu\left(Z_{n}\right)-\mu\left(Z_{n+1}\right) .
$$

Therefore $\sum_{n=1}^{\infty} \mu\left(Y_{n}\right) \leq \mu\left(Z_{1}\right)=\mu\left(f^{-1}(Y)\right)=\mu(Y)$.

Markov partitions. Let $f \in \mathcal{E}_{0}^{\text {lip }}$. The points in $f^{-n}(0)$ divide the circle into $\left|d_{f}\right|^{n}$ open intervals, which are called Markov intervals of order $n$. The image of a Markov interval of order $n$ is a Markov interval of order $n-1$. If $I$ is a Markov interval of order $n$ then $f^{n} \mid I$ is a $1-1$ map onto $(0,1)$.

If $I$ is a Markov interval and $f_{I}$ is the first return map to $I$, then there exist disjoint (Markov) intervals $I_{j} \subset I$ such that $f_{I} \mid I_{j}$ is an homeomorphism onto $I$ for each $j$. If $f$ is conservative with respect to Lebesgue measure then the intervals $I_{j}$ cover $I$ $m$-mod 0 .

Piecewise linear approximations. Let us say that a map $f \in \mathcal{E}_{0}^{\text {lip }}$ belongs to $\mathcal{E}_{0}^{\text {pl }}$ if there exists $n \geq 1$ such that for every Markov interval $I$ of order $n, f \mid I$ is linear.

Lemma 2. The set $\mathcal{E}_{0}^{\mathrm{pl}}$ is dense in $\mathcal{E}_{0}^{1}$ in the Lipschitz metric.

Proof. Given $f \in \mathcal{E}_{0}^{1}$ and $n \geq 2$, we define a map $f_{n}: \mathbb{T}^{1} \rightarrow \mathbb{T}^{1}$ as follows: For each Markov interval $I$ of order $n$ for $f$, let $f_{n}$ map $I$ onto $f(I)$ linearly, and so that $f_{n}$ equals $f$ in the boundary of $I$. Clearly, $f_{n} \in \mathcal{E}_{0}^{\mathrm{pl}}$. We claim that $f_{n} \rightarrow f$ in the Lipschitz metric.

Notice that the lengths of Markov intervals $I$ of order $n$ go uniformly to 0 as $n \rightarrow \infty$ : in fact, $m(I) \leq \lambda_{f}^{-n}$. Since $f$ is $C^{1}$, for every $\delta>0$ we can choose $n_{0}$ such that in each Markov interval $I$ of order $n \geq n_{0}$, $\sup _{x, y \in I}\left|f^{\prime}(x)-f^{\prime}(y)\right| \leq \delta$. Then $d_{\text {lip }}\left(f, f_{n}\right) \leq \delta$.

Lemma 3. Every $f \in \mathcal{E}_{0}^{\mathrm{pl}}$ is ergodic and conservative with respect to Lebesgue measure.

Proof. Given $f \in \mathcal{E}_{0}^{\mathrm{pl}}$, let $n$ be such $f$ is linear on Markov intervals of order $n$. Let $X$ be a forward invariant set of positive Lebesgue measure. Notice that if $I$ is a Markov interval of order $N \geq n$, then $f^{N-n} \mid I$ is linear onto some Markov interval of order $n$, and $f^{N} \mid I$ is onto $(0,1)$ with distortion bounded by $\Lambda_{f}^{n} / \lambda_{f}^{n}$. By the Lebesgue Density Points theorem, for almost every $x \in X, \lim _{N \rightarrow \infty} m\left(I_{N} \cap X\right) / m\left(I_{N}\right)=1$, where $I_{N}$ is the Markov interval of order $N$ containing $x$. Applying $f^{N}$ and using the bound on the distortion, we see that $m\left(f^{N}\left(X \cap I_{N}\right)\right) \rightarrow 1$ as $N \rightarrow \infty$. So $m(X)=1$ and the result follows. 


\section{Plan of proof}

Definition 1. Let $I$ be an interval and $\phi: I \rightarrow I$ non-singular (w.r.t. the Lebesgue measure) map. Then $\phi$ is called distorted if there exists a measurable set $A \subset I$ such that $\frac{m(A)}{m(I)}>.4$, and $\frac{d\left(\phi_{*} m_{I}\right)}{d m_{I}}>2$ on $A$ (where $m_{I}$ is Lebesgue measure on $I$ ).

Recall that

$$
\frac{d\left(\phi_{*} m_{I}\right)}{d m_{I}}(y)=\sum_{x \in f^{-1}(y)} \frac{1}{\left|\phi^{\prime}(x)\right|} .
$$

Definition 2. If $\delta>0$ and $f \in \mathcal{E}_{0}^{\text {lip }}$ then $f$ is called $\delta$-good if there exists a family $\mathcal{I}$ of Markov intervals (possibly of different orders), all of length at most $\delta$, such that $m\left(\bigcup_{I \in \mathcal{I}} I\right)>1-\delta$ and for every $I \in \mathcal{I}$, the first return map $f_{I}: I \rightarrow I$ is distorted.

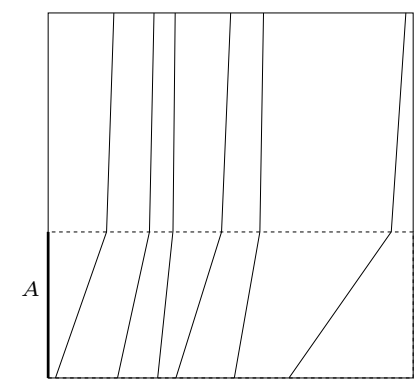

Figure 1. A typical graph of a distorted $f_{I}$. In this example, $A$ is an interval. There should be infinitely many branches.

We can now state the three key technical results of this paper.

Proposition 1. If $f \in \mathcal{E}_{0}^{1}$ is $\delta$-good for every $\delta>0$ then $f$ has no acio.

The next proposition says the condition of being $\delta$-good is open in two senses.

Proposition 2. Let $f \in \mathcal{E}_{0}^{\mathrm{lip}}$ be $\delta$-good for some $\delta>0$. Then:

(i) There exists $\beta>0$ such that if a map $\tilde{f} \in \mathcal{E}_{0}^{\text {lip }}$ satisfies

$$
m\left(\left\{x \in \mathbb{T}^{1} ; \tilde{f}(x) \neq f(x)\right\}\right)<\beta
$$

then it is $\delta$-good.

(ii) Assuming that $f \in \mathcal{E}_{0}^{1}$, there exists $\gamma>0$ such that if $\tilde{f} \in \mathcal{E}_{0}^{1}$ satisfies $d_{\text {lip }}(\tilde{f}, f)<\gamma$ then $\tilde{f}$ is $\delta$-good.

Proposition 3. For any $\delta>0$, the set of maps that are $\delta$-good is dense in $\mathcal{E}_{0}^{1}$ with the Lipschitz metric.

Let us first see how to conclude theorem 2 (and hence, by the reduction, theorem 1) from the three propositions.

Proof of theorem 2. The set $\mathcal{U}_{\delta}$ of $f \in \mathcal{E}_{0}^{1}$ which are $\delta$-good is $C^{1}$-open, by part (ii) of proposition 2, and $C^{1}$-dense, by proposition 3. So $\mathcal{R}_{0}=\bigcap_{\delta>0} \mathcal{U}_{\delta}$ is a residual set of $\mathcal{E}_{0}^{1}$, and by proposition 1 it consists of maps which do not have an aci $\sigma$. 
We now prove propositions 1 and 2, and leave the harder proof of proposition 3 (where part (i) in proposition 2 is used) for the next section.

Proof of proposition 1. Assume that for all $\delta>0, f \in \mathcal{E}^{1}$ is $\delta$-good; let $\mathcal{I}_{\delta}$ be the corresponding family of intervals as in definition 2. Assume $f$ has an aci $\sigma \mu$, and let $\rho=\frac{d \mu}{d m}$ be its density. Let $c>0$ be such that the set $Z=\left\{x \in \mathbb{T}^{1} ; c \leq \rho(x) \leq 1.1 c\right\}$ has positive Lebesgue measure. By a density point argument, there exists an interval $I \in \bigcup_{\delta} \mathcal{I}_{\delta}$ such that $m(I \cap Z) / m(I)>.9$. Since $f_{I}$ is distorted, there exists $A \subset I$ such that $m(A) / m(I)=.4$ and $d\left(f_{I}\right)_{*} m / d m>2$ on $A$. Let $Y=A \cap Z$. We have

$$
m(Y) \geq m(A)-m(I \backslash Z)>.4 m(I)-.1 m(I)=.3 m(I)
$$

and

Moreover,

$$
\mu(Y) \leq 1.1 \mathrm{~cm}(Y) \leq 1.1 \times .4 \mathrm{~cm}(I)=.44 \mathrm{~cm}(I)
$$

$$
\begin{aligned}
\mu\left(f_{I}^{-1}(Y)\right) \geq c m & \left(Z \cap f_{I}^{-1}(Y)\right) \geq c\left[m\left(f_{I}^{-1}(Y)\right)-m(I \backslash Z)\right] \\
& >c[2 m(Y)-.1 m(I)] \geq c[2 \times .3-.1] m(I)=.5 c m(I)>\mu(Y) .
\end{aligned}
$$

This contradicts lemma 1.

Proof of proposition 2. Let $\mathcal{I}$ be the family of Markov intervals as in definition 2; clearly we can assume it is finite, say, $\mathcal{I}=\left\{I_{i} ; 1 \leq i \leq i_{0}\right\}$. Let $A_{i} \subset I_{i}$ be the set that gets enlarged under $\left(f_{I_{i}}\right)^{-1}$ according to definition 1 . Let $J_{i, 1}, J_{i, 2}, \ldots$ be the connected components of the domain of $f_{I_{i}}$, and let $n_{i, j}$ be such that $f^{n_{i, j}}\left(J_{i, j}\right)=I_{i}$. Then

$$
\sum_{j=1}^{\infty}\left|\left(\left(f^{n_{i, j}} \mid J_{i, j}\right)^{-1}\right)^{\prime}(y)\right|>2 \quad \text { for every } y \in A_{i} .
$$

Slightly reducing the sets $A_{i}$ (still keeping $m\left(A_{i}\right) / m\left(I_{i}\right)>.4$ ), we can find $j_{0}$ such that

$$
\sum_{j=1}^{j_{0}}\left|\left(\left(f^{n_{i, j}} \mid J_{i, j}\right)^{-1}\right)^{\prime}(y)\right|>2+\varepsilon \quad \text { for every } y \in A_{i}, \text { for every } i=1, \ldots, i_{0},
$$

where $\varepsilon$ is some fixed positive number. Also let $N=\max \left\{n_{i, j} ; 1 \leq i \leq i_{0}, 1 \leq j \leq\right.$ $\left.j_{0}\right\}$.

If $\tilde{f}$ is another map in $\mathcal{E}_{0}^{\text {lip }}$ which is $C^{0}$-close to $f$, then for each interval $I_{i} \in \mathcal{I}$ there is an interval $\tilde{I}_{i}$ which is Markov for $\tilde{f}$ and is close to $I_{i}$. Clearly if the $C^{0}$-distance between $\tilde{f}$ and $f$ is sufficiently small then each $\tilde{I}_{i}$ has length $<\delta$, and their union has measure $>1-\delta$. Further, for each $\tilde{I}_{i}$ there exist intervals $\tilde{J}_{i, j}, 1 \leq j \leq j_{0}$, which are close to $J_{i, j}$ and such that $\tilde{f}^{n_{i, j}}\left(\tilde{J}_{i, j}\right)=\tilde{I}_{i}$.

With these notations fixed, we complete the proofs of the two parts of the proposition separately.

Part (i): Let $\tilde{f} \in \mathcal{E}_{0}^{\text {lip }}$ so that the set $U=\left\{x \in \mathbb{T}^{1} ; \tilde{f}(x) \neq f(x)\right\}$ has $m(U)<\beta$, where how small $\beta$ needs to be will become clear along the way. First, notice that the $C^{0}$-distance between $\tilde{f}$ and $f$ is small (in fact, less than $\Lambda_{f} \beta$ ). So we can define intervals $\tilde{I}_{i}, \tilde{J}_{i, j}$, for $1 \leq i \leq i_{0}$ and $1 \leq j \leq j_{0}$ as explained above. Let $V=$ $\bigcup_{n=0}^{N} f^{n}(U)$. Then $m(V) \leq\left(1+\Lambda_{f}+\cdots+\Lambda_{f}^{N}\right) \beta$ is small. Define $\tilde{A}_{i}=\tilde{I}_{i} \cap A_{i} \backslash V$. Then $m\left(A_{i} \backslash \tilde{A}_{i}\right)$ is small: at most $m\left(I_{i} \backslash \tilde{I}_{i}\right)+m(V)$. So if $\beta$ is small enough then 
$m\left(\tilde{A}_{i}\right) / m\left(\tilde{I}_{i}\right)>.4$. Moreover, if $y \in \tilde{A}_{i}$ then $\frac{d\left(\tilde{f}_{\tilde{I}_{i}}\right)_{*} m}{d m}(y)>2+\varepsilon$. This shows that $\tilde{f}_{\tilde{I}_{i}}$ is distorted for each $i$ and accordingly that $\tilde{f}$ is $\delta$-good.

Part (ii): Now assume $f$ is $C^{1}$ and $\tilde{f}$ is $\gamma-C^{1}$-close to $f$. Again we can define intervals $\tilde{I}_{i}, \tilde{J}_{i, j}$, for $1 \leq i \leq i_{0}$ and $1 \leq j \leq j_{0}$. Let $\tilde{A}_{i}=\tilde{I}_{i} \cap A_{i}$. By taking a small $\gamma$, we guarantee that $\tilde{f}^{n}$ is $C^{1}$-close to $f^{n}$ for $1 \leq n \leq N$, and therefore

$$
\sum_{j=1}^{j_{0}}\left|\left(\left(\tilde{f}^{n_{i, j}} \mid \tilde{J}_{i, j}\right)^{-1}\right)^{\prime}(y)\right|>2 \quad \text { for every } y \in \tilde{A}_{i} .
$$

So the $\tilde{f}_{\tilde{I}_{i}}$ are distorted and and $\tilde{f}$ is $\delta$-good.

We remark that with a little more effort it is possible to improve simultaneously the two parts of proposition 2 , showing that being $\delta$-good is an open condition in $\mathcal{E}_{0}^{\text {lip }}$ in the total variation metric $d_{\mathrm{BV}}(f, g)=\int_{\mathbb{T}^{1}}\left|f^{\prime}-g^{\prime}\right| d m$.

\section{Proof of proposition 3}

Let $f_{0} \in \mathcal{E}_{0}^{1}$ and $\delta>0$; we will show that there exists a $\delta$-good map $h \in \mathcal{E}_{0}^{1}$ such that $d_{\text {lip }}\left(h, f_{0}\right)<3 \delta$. For simplicity, we will assume that $f$ is orientation-preserving. The proof can be easily adapted to cover the general case.

Step 1. Linearization. By lemma 2, we can find $f \in \mathcal{E}_{0}^{\mathrm{pl}}$ with $d_{\text {lip }}\left(f, f_{0}\right)<\delta$. Since $f \in \mathcal{E}_{0}^{\mathrm{pl}}$, there exists $n_{0}$ such that if $I$ is a Markov interval of order $n \geq n_{0}$ then $f \mid I$ is linear.

Let $\ell \geq n_{0}$ be large (to be specified later). Fix a Markov interval $T$ such that the sets $T, f^{-1}(T), \ldots, f^{-\ell}(T)$ are disjoint, and their union has Lebesgue measure less than $\delta$.

Let $\mathcal{P}_{T}$ be the collection of (Markov) subintervals of $T$ that are sent onto $T$ by $f_{T}$. Let $K_{T}=T \backslash \bigcup_{I \in \mathcal{P}_{T}} I$. Notice that for any $I \in \mathcal{P}_{T}$, $\operatorname{order}(I) \geq \operatorname{order}(T)+\ell$, $\operatorname{order}(T) \geq \ell \geq n_{0}$ and $f_{T}\left|I=f^{\operatorname{order}(I)-\operatorname{order}(T)}\right| I$ is linear.

Step 2. Another perturbation. If $I$ is an interval, denote by $\Phi_{I}$ the only orderpreserving linear bijection $I \rightarrow(0,1)$.

Each interval in $\mathcal{P}_{T}$ has length at most $\lambda_{f}^{-\ell} m(T)$. Since $\ell$ is large, we can find $\xi \in T \backslash \bigcup_{I \in \mathcal{P}_{T}} I$ such that

$$
\eta=\Phi_{T}(\xi) \in(.4, .41) .
$$

We define a perturbation $g$ of $f$ as follows:

- $g$ equals $f$ outside $f^{-1}\left(T \backslash K_{T}\right) \cup \cdots \cup f^{-\ell}\left(T \backslash K_{T}\right)$.

- Let $\Xi=f_{T}^{-1}(\xi)$. Consider all sequences of intervals

$$
I_{\ell} \rightarrow I_{\ell-1} \rightarrow \cdots \rightarrow I_{0}
$$

with $f\left(I_{j}\right)=I_{j-1}$ and $I_{0} \in \mathcal{P}_{T}$. Let $\xi_{0}=I_{0} \cap \Xi$. Then $\Phi_{I_{0}}\left(\xi_{0}\right)=\eta$. Let $\xi_{i}=$ $\Phi_{I_{i}}^{-1}\left(\eta+\frac{i}{2 \ell}\right)$. We define $g \mid I_{i}$ for $1 \leq i \leq \ell$ as the unique orientation preserving homeomorphism onto $I_{i-1}$ whose restriction to each connected component of $I_{i} \backslash\left\{\xi_{i}\right\}$ is linear and such that $g\left(\xi_{i}\right)=\xi_{i-1}$. Let $Q=\Phi_{I_{0}} \circ g^{\ell} \circ \Phi_{I_{\ell}}^{-1}$. It is the homeomorphism of $(0,1)$ depicted in figure 2 .

Notice that the Lipschitz distance between $g$ and $f$ is at most $C_{f} / \ell$, for some constant $C_{f}$ depending on $f$ only, and hence it is $<\delta$ since $\ell$ is large. 


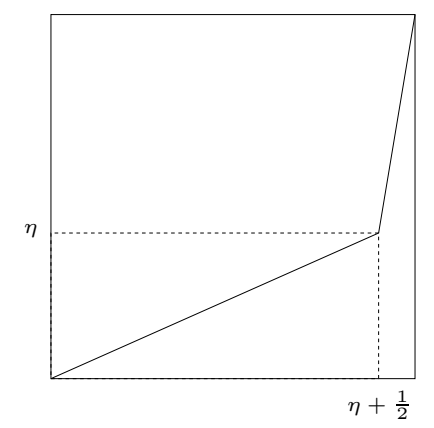

Figure 2. Graph of $Q:(0,1) \rightarrow(0,1)$.

Step 3. Some properties of $g$. Let $\mathcal{P}_{g, T}^{k}$ be the family of ( $g$-Markov) subintervals of $T$ that are sent onto $T$ by $g_{T}^{k}$. Notice that $\mathcal{P}_{g, T}^{1}=\mathcal{P}_{T}$.

If $\zeta$ is a singularity for $g$, in the sense that $g$ is not linear in a neighborhood of $\zeta$, then:

(i) either $\zeta$ is a singularity for $f$ - in this case it is contained in $f^{-n_{0}}(0)$;

(ii) or $\zeta$ belongs to $f^{-i}\left(K_{T}\right)$ for some $1 \leq i \leq \ell$;

(iii) or $\zeta$ belongs to $g^{-i}(\Xi)$ for some $1 \leq i \leq \ell$.

Lemma 4. If $L$ is an element of $\mathcal{P}_{g, T}^{2}$ and $J$ is a connected component of $g^{-k}(L)$, $k \geq 1$, then $g^{k} \mid J$ is linear.

Proof. If $g^{k} \mid J$ is not linear then $g^{j}(J)$ intersects a singularity $\zeta$ of $g$ for some $0 \leq j<k$. Since $L=g^{k}(J)$ belongs to $\mathcal{P}_{g, T}^{2}$, there are at least three different $i>0$ such that $g^{i}(\zeta) \in T$. Thus $\zeta$ cannot be of the type (i) above: indeed, the set $f^{-n_{0}}(0)=g^{-n_{0}}(0)$ is forward invariant for both $f$ and $g$, and it does not intersect $T$. $\zeta$ cannot be of the type (ii): the first iterate of $\zeta$ that belongs to $T$ belongs indeed to $K_{T}$, and subsequent iterates do not enter $T$ again. $\zeta$ cannot be a singularity of type (iii): the first iterate of $\zeta$ that belongs to $T$ also belongs to $\Xi$, so the second iterate that belongs to $T$ is $\xi$, and the subsequent iterates lie outside $T$. So there can be no such singularity, and the result follows.

Lemma 5. If $L$ is an element of $\mathcal{P}_{g, T}^{k}$ then $\operatorname{Dist}\left(g_{T}^{k} \mid L\right) \leq\left(\Lambda_{g} / \lambda_{g}\right)^{2 \ell}$.

Proof. Take $L \in \mathcal{P}_{g, T}^{1}$. Let $r$ be such that $g^{r}(L)=T$. Then $g^{r-\ell} \mid L$ is linear, and hence $\operatorname{Dist}\left(g_{T} \mid L\right)=\operatorname{Dist}\left(g^{\ell} \mid g^{r-\ell}(L)\right) \leq\left(\Lambda_{g} / \lambda_{g}\right)^{\ell}$. This implies the assertion of the lemma for $k=1$ and $k=2$. Now, if $k \geq 2$ and and $L \in \mathcal{P}_{g, T}^{k}$ then, by lemma 4 , $g_{T}^{k-2} \mid L$ is linear, so the assertion also follows.

Lemma 6. $g$ is ergodic and conservative with respect to Lebesgue measure.

Proof. We will adapt the argument in the proof of lemma 3 . Let $X \subset \mathbb{T}^{1}$ be a forward $g$-invariant set with $m(X)>0$.

Assume that $X \cap T$ has zero Lebesgue measure. By a density point argument, we can take a $g$-Markov interval $L$ such that $m(L \cap X) / m(L)$ is close to 1 . Any forwardimage $g^{k}(L)$ cannot be contained in the set $W=\bigcup_{j=1}^{\ell} f^{-j}(T)$. By the Markov 
property, $g^{k}(L) \cap W=\varnothing$ for $0 \leq k \leq \operatorname{order}(L)-\operatorname{order}(T)-\ell$. Since $g$ equals $f$ outside $W, g^{\operatorname{order}(L)-\operatorname{order}(T)-\ell} \mid L$ is linear. In particular,

$$
\operatorname{Dist}\left(g^{\operatorname{order}(L)} \mid L\right) \leq\left(\Lambda_{g} / \lambda_{g}\right)^{\operatorname{order}(T)+\ell} .
$$

It follows that $m\left(g^{\operatorname{order}(L)}(L \cap X)\right)$ is close to 1 . This shows that the assumption $m(X \cap T)=0$ cannot be true.

Since $f$ is conservative (lemma 3 ), $m$-almost every point in $T$ returns to $T$ by forward iterates of $f$. It follows that the same is true for $g$. So the intervals in $\mathcal{P}_{g, T}^{k}$ cover $T m$-mod 0 . Moreover, these intervals have lengths at most $\lambda_{g}^{k \ell}$. Therefore we can find $L$ and $k \geq 2$ such that $L \in \mathcal{P}_{g, T}^{k}$ and $m(L \cap X) / m(L)$ is arbitrarily close to 1. By lemma $5, m\left(g_{T}^{k}(L \cap X)\right) / m(T)$ is arbitrarily close to 1 . It follows that $m(X \cap T) / m(T)=1$. So $g^{\operatorname{order}(T)}(X \cap T) \subset X$ has full $m$-measure on the circle.

Lemma 7. $g$ is $\delta$-good.

Proof. First, let us define the family $\mathcal{I}$ : an interval $I$ belongs to $\mathcal{I}$ iff there exists $n=n(I)>\ell$ such that $f^{n}(I)=T$ and $f^{k}(T) \cap T=\varnothing$ for $0 \leq k<n$. Notice that:

- For every $I \in \mathcal{I}, g^{k}(I)=f^{k}(I)$ for $0 \leq k \leq n(I)$, and hence $I$ is Markov for $g$.

- For every $I \in \mathcal{I}, m(I)<m(T)<\delta$.

- $\bigcup_{I \in \mathcal{I}} I=\mathbb{T}^{1} \backslash \bigcup_{j=0}^{\ell} f^{-j}(T) m$-mod 0 ; in particular $m\left(\bigcup_{I \in \mathcal{I}} I\right)>1-\delta$.

We have to show that for each $I \in \mathcal{I}, g_{I}$ is distorted. For this, it is enough to prove that (where $\mathcal{P}_{g, I}$ is the collection of intervals $J \subset I$ that are sent onto $I$ by $g_{I}$ ):

(i) $\bigcup_{J \in \mathcal{P}_{g, I}} J=I m$-mod 0 ;

(ii) if $J \in \mathcal{P}_{g, I}$ and $r$ is such that $g^{r}(J)=I$ then $\Phi_{I} \circ g^{r} \circ \Phi_{J}^{-1}=Q$.

Indeed in this case we can take $A=\Phi_{I}^{-1}(0, \eta)$ in definition 1 .

The first property follows from lemma 6 . Let us check the second one. Let $\left\{i_{1}<\right.$ $\left.\cdots<i_{t}\right\}=\left\{k ; 0 \leq k \leq r, g^{k}(J) \subset g^{-\ell}(T)\right\}$. Then

$$
0<i_{1}<i_{1}+\ell<\cdots<i_{t-1}<i_{t-1}+\ell<i_{t}<i_{t}+\ell<r .
$$

We claim that $g^{i_{t}} \mid J$ is linear. This is clear if $t=1$. Notice that $g^{i_{t}+\ell}(J)$ is an element of $\mathcal{P}_{T}$ : indeed, $g^{r-i_{t}-\ell+n(I)}$ takes $g^{i_{t}+\ell}(J)$ onto $T$. This implies that $g^{i_{j}+\ell}(J)$ is an element of $\mathcal{P}_{g, T}^{1+t-j}$. By lemma $4, g^{i_{t-1}+\ell} \mid J$ is linear, therefore $g^{i_{t}} \mid J$ is linear, as claimed.

Since $g^{i_{t}+\ell}(J)$ is an element of $\mathcal{P}_{T}, \Phi_{g^{i_{t}+\ell}(J)} \circ g^{\ell} \circ \Phi_{g^{i_{t}(J)}}^{-1}=Q$. It is also clear that $g^{r-i_{t}-\ell} \mid g^{i_{t}+\ell}(J)$ is linear. It follows that $\Phi_{I} \circ g^{r} \circ \Phi_{J}^{-1}=Q$, as desired.

Step 4. Smoothening $g$. For $X \subset \mathbb{T}^{1}$ and $\varepsilon>0$, let $B_{\varepsilon}(X)$ be the $\varepsilon$-neighborhood of $X$. Let $S$ be the (already described) set of singularities of $g$; then $S$ is closed in $\mathbb{T}^{1}$ and $m(S)=0$. In particular, $m\left(B_{\alpha}(S)\right) \rightarrow 0$ as $\alpha \rightarrow 0$.

Let $G: \mathbb{R} \rightarrow \mathbb{R}$ be the lift of $g$ satisfying $G(0)=0$. For $\alpha>0$, let

$$
G_{\alpha}(x)=\frac{1}{2 \alpha} \int_{x-\alpha}^{x+\alpha} G(y) d y .
$$

Then $G_{\alpha}: \mathbb{R} \rightarrow \mathbb{R}$ is the lift of some $C^{1}$ map $g_{\alpha}: \mathbb{T}^{1} \rightarrow \mathbb{T}^{1}$ such that for every $x \in \mathbb{T}^{1}$,

$$
\left|g_{\alpha}^{\prime}(x)-f_{0}^{\prime}(x)\right| \leq d_{\operatorname{lip}}\left(g, f_{0}\right)+\sup _{y \in B_{\alpha}(x)}\left|f_{0}^{\prime}(y)-f_{0}^{\prime}(x)\right| .
$$


(Recall $f_{0}$ is the original $C^{1}$ map.) Hence $\left|g_{\alpha}^{\prime}(x)-f_{0}^{\prime}(x)\right| \leq 2 \delta$ for every $x \in \mathbb{T}^{1}$ provided $\alpha$ is sufficiently small. Moreover, since $g$ is linear on connected components of $\mathbb{T}^{1} \backslash S, g_{\alpha}$ equals $g$ outside the $\alpha$-neighborhood of $S$.

Unfortunately, $g_{\alpha}$ does not necessarily fix 0 . To remedy that, take a family of $C^{1}$ diffeomorphisms $\phi_{\xi}: \mathbb{T}^{1} \rightarrow \mathbb{T}^{1}$ parameterized by $\xi \in \mathbb{T}^{1}$, such that

$$
\phi_{\xi}(\xi)=0, \quad \lim _{\xi \rightarrow 0} \sup _{x \in \mathbb{T}^{1}}\left|\phi_{\xi}^{\prime}(x)-1\right|=0, \quad \text { and } \quad \lim _{\xi \rightarrow 0} m\left\{x \in \mathbb{T}^{1} ; \phi_{\xi}(x) \neq x\right\}=0 .
$$

Define $h_{\alpha}=\phi_{g_{\alpha}(0)} \circ g_{\alpha}$. If $\alpha$ is small then $h_{\alpha} \in \mathcal{E}_{0}^{1}$ and $d_{\text {lip }}\left(h_{\alpha}, f_{0}\right)<3 \delta$. Also, $\lim _{\alpha \rightarrow 0} m\left\{x \in \mathbb{T}^{1} ; h_{\alpha}(x) \neq g(x)\right\}=0$. By part (i) of proposition $2, h_{\alpha}$ is $\delta$-good provided $\alpha$ is small enough. This concludes the proof of proposition 3.

\section{Acknowledgements}

This research was partially conducted during the period A.A. served as a Clay Research Fellow. The paper was written while J.B. was visiting IMPA, partially supported by Procad/CAPES project. J.B. thanks Philippe Thieullen for providing several references and for stimulating conversations.

\section{References}

[1] A. Avila and J. Bochi, A generic $C^{1}$ map has no absolutely continuous invariant probability measure, Nonlinearity 19 (2006), no. 11, 2717-2725.

[2] H. Bruin and J. Hawkins, Examples of expanding $C^{1}$ maps having no $\sigma$-finite invariant measure equivalent to Lebesgue, Israel J. Math. 108 (1998), 83-107.

[3] J. T. Campbell and A. N. Quas, A generic $C^{1}$ expanding map has a singular $S-R-B$ measure, Commun. Math. Phys. 221 (2001), no. 2, 335-349.

[4] P. R. Halmos, Invariant measures, Ann. of Math. 48 (1947), 735-754.

[5] T. Hamachi, On a Bernoulli shift with non-identical factor measures, Ergodic Theory Dyn. Syst. 1 (1981), no. 3, 273-283.

[6] J. Hawkins and C. E. Silva, Noninvertible transformations admitting no absolutely continuous $\sigma$-finite invariant measure, Proc. Amer. Math. Soc. 111 (1991), no. 2, 455-463.

[7] M. R. Herman, Sur la conjugaison différentiable des difféomorphismes du cercle à des rotations, Publ. Math. IHES 49 (1979), 5-233.

[8] M. V. Jakobson, Smooth mappings of the circle into itself, Mat. Sb. (N.S.) 85 (127) (1971), $163-188$.

[9] Y. Katznelson, Sigma-finite invariant measures for smooth mappings of the circle, J. Analyse Math. 31 (1977), 1-18.

[10] O. Kozlovski, W. Shen, and S. van Strien, Density of hyperbolicity in dimension one, Ann. of Math.. To appear.

[11] R. Mañé, Hyperbolicity, sinks and measure in one-dimensional dynamics, Comm. Math. Phys. 100 (1985), no. 4, 495-524.

[12] D. S. Ornstein, On invariant measures, Bull. Amer. Math. Soc. 66 (1960), 297-300.

[13] A. Quas, Most expanding maps have no absolutely continuous invariant measure, Studia Math. 134 (1999), no. 1, 69-78.

CNRS UMR 7599, Laboratoire de Probabilités et Modèles aléatoires.,

Université Pierre et Marie Curie, Boîte courrier 188. 75252-Paris Cedex 05, France

$U R L$ : www.proba.jussieu.fr/pageperso/artur/

E-mail address: artur@ccr.jussieu.fr

Instituto de Matemática, UfRGs, Porto Alegre, Brazil

$U R L$ : www.mat.ufrgs.br/ jairo

E-mail address: jairo@mat.ufrgs.br 